\title{
Synthesis and Application of Histidine-Modified Poly(Glycidyl Methacrylate/Ethylene Glycol Dimethacrylate) Sorbent for Isolation of Caffeine from Black and Green Tea Samples
}

\author{
Florian Meischl ${ }^{1} \cdot$ Klemens Losso $^{1} \cdot$ Christian G. Kirchler $^{1}$ - Stefan E. Stuppner ${ }^{1}$. Christian W. Huck ${ }^{1}$. Matthias Rainer ${ }^{1}$
}

Received: 17 May 2018 / Revised: 21 August 2018 / Accepted: 27 August 2018 / Published online: 1 September 2018

(c) The Author(s) 2018

\begin{abstract}
This work presents the synthesis of a polymeric mixed-mode solid-phase extraction (SPE) sorbent for clean-up and isolation of caffeine from black and green tea samples. The material was synthesized by a simple thermally initiated copolymerization of glycidyl methacrylate and ethylene glycol dimethacrylate. Further functionalization was executed with histidine (HIS). Functional groups were investigated by attenuated total-reflection infrared spectroscopy. Furthermore, nitrogen sorption porosimetry was executed and revealed surface areas of $90 \mathrm{~m}^{2} \mathrm{~g}^{-1}$. Adsorption capacities for caffeine were compared between functionalized and non-modified polymers and showed maximum capacities of 3.01 and $4.82 \mathrm{mg} \mathrm{g}^{-1}$ polymer, respectively. Time adsorption profiles revealed an equilibrium adsorption after $15 \mathrm{~min}$. The proposed polymer was used for SPE of black and green tea extracts and showed excellent clean-up efficiency for isolation of caffeine with recoveries ranging from 89 to $93 \%$. When compared to commercially available Oasis HLB, the HIS-functionalized polymer demonstrated a distinctly better performance for clean-up. Finally, the proposed method was validated regarding international (ICH) guidelines and regulations.
\end{abstract}

Keywords Caffeine $\cdot$ Histidine $\cdot$ Polymer modification $\cdot$ Solid-phase extraction $\cdot$ Tea

\begin{tabular}{|c|c|}
\hline \multicolumn{2}{|c|}{ Abbreviations } \\
\hline $\mathrm{ACN}$ & Acetonitrile \\
\hline AIBN & Azobisisobutyronitrile \\
\hline ATR-IR & $\begin{array}{l}\text { Attenuated total-reflection infrared } \\
\text { spectroscopy }\end{array}$ \\
\hline DVB & Divinylbenzene \\
\hline EGDMA & Ethylene glycol dimethacrylate \\
\hline FT-IR & Fourier-transform infrared spectroscopy \\
\hline GMA & Glycidyl methacrylate \\
\hline HIS & Histidine \\
\hline HPLC & High-performance liquid chromatography \\
\hline H-PTFE & Hydrophilized polytetrafluoroethylene \\
\hline $\mathrm{ICH}$ & International Conference on Harmonization \\
\hline
\end{tabular}

Electronic supplementary material The online version of this article (https://doi.org/10.1007/s10337-018-3601-6) contains supplementary material, which is available to authorized users.

Matthias Rainer

m.rainer@uibk.ac.at

1 Institute of Analytical Chemistry and Radiochemistry, Leopold-Franzens University of Innsbruck, Innrain 80-82, 6020 Innsbruck, Austria

$\begin{array}{ll}\text { LOD } & \text { Limit of detection } \\ \text { LOQ } & \text { Limit of quantitation } \\ \text { MeOH } & \text { Methanol } \\ \text { RSD } & \text { Relative standard deviation } \\ \text { SPE } & \text { Solid-phase extraction } \\ \text { UV } & \text { Ultraviolet } \\ \text { VWD } & \text { Variable wavelength detector }\end{array}$

\section{Introduction}

Plant extracts exist in abundance of active components and their analysis is very often difficult [1]. Nowadays, different methods and techniques have been developed for qualitative and quantitative evaluation of such complex mixtures. A well-established sample preparation procedure for herbal material is solid-phase extraction (SPE) [2, 3]. It is used either for clean-up or concentration of liquid samples. Solid sorbents are normally placed in cartridges with amounts varying from $10 \mathrm{mg}$ to $10 \mathrm{~g}$. A typical SPE protocol can be divided into four essential steps: after conditioning the sorbent (1) the plant extract is forced through the sorbent material (2). Analytes can bind by same mechanisms as described 
in liquid chromatography, i.e., dipole-dipole interactions, hydrogen bonding, hydrophobic and ionic interactions [4-6]. After sample loading interfering compounds are removed using several washing solvents (3) and finally target analytes are eluted from the sorbent (4). Alternatively it is also possible to elute target compounds without performing prior washing steps $[7,8]$.

The application of polymeric sorbents in SPE has significantly increased in the last years due to their broad applicability and distinct advantages in comparison to silica-based materials. Most of them are highly crosslinked materials with a hydrophobic backbone through incorporation of divinylbenzene (DVB). The usage of various monomer types like vinyl or allyl compounds enhances the broad applicability of these polymers in SPE [9-12].

A widely used monomer is glycidyl methacrylate (GMA) which is formed by condensation of methacrylic acid and glycidol. It forms so-called epoxy polymers with possibility for further modification after base-catalyzed ring opening of the epoxide. Industry developed several applications for these materials acting like adhesives, coatings, composites or sorbents for bioanalysis. Main advantages for their usage are that no emissions of volatile products occur during polymerization and their characteristics for introduction of new functionalities [13, 14].

In this work, a copolymer was synthesized using GMA as monomer and ethylene glycol dimethacrylate (EGDMA) as hydrophilic crosslinker. Polymerization was performed by a thermally initiated precipitation polymerization procedure. Further functional modification was executed using L-histidine (HIS). This basic $\alpha$-amino acid consisting of an $\alpha$-amino group, an imidazole side chain and a carboxylic acid side chain is used in the bioanalysis of proteins. At a physiological $\mathrm{pH}$ value HIS is a positively charged amino acid. In the body HIS also converts into further biological active amines like histamine which plays an important role in immune response and inflammation [15, 16]. In the literature, HIS modification was already proven to act as photoreactive polymer [17], block copolymers [18] or imprinted polymeric beads [19].

The modified sorbent was comprehensively characterized by infrared spectroscopy and nitrogen sorption porosimetry. A capacity study was further executed preliminary to solidphase extraction. Isolation of caffeine from green and black tea extracts was performed by development of a preferably selective extraction process using the self-fabricated polymeric material.

\section{Materials and Methods}

\section{Reagents and Materials}

Azobisisobutyronitrile (AIBN, purum, $\geq 98 \%$ ), ethylene glycol dimethacrylate (EGDMA, 99\%), glycidyl methacrylate (GMA, $\geq 97 \%$ ), L-histidine monohydrochloride monohydrate (HIS, $\geq 97 \%$ ) and caffeine ( $\geq 99 \%)$ were purchased from Sigma-Aldrich (Buchs, Switzerland). Sodium carbonate ( $\geq 99 \%$ ) was obtained from Carl Roth (Karlsruhe, Germany). Acetonitrile (ACN) and methanol $(\mathrm{MeOH})$ were all HPLC grade (Chromasolv) and also from Sigma-Aldrich. Water was obtained from a Milli-Q water purification system from Merck Millipore (Billerica, Massachusetts).

Empty polypropylene SPE tubes $(1 \mathrm{~mL})$ with polyethylene frits (porosity $20 \mu \mathrm{m}$ ) were ordered from Sigma-Aldrich and Carl Roth. The SPE sorbent Oasis HLB $\left(1 \mathrm{~cm}^{3}, 30 \mathrm{mg}\right)$ was acquired from Waters Corp. (Milford, Massachusetts, USA). Hydrophilized polytetrafluoroethylene (H-PTFE) filters were purchased from Machery Nagel (Düren, Germany).

Black and green tea samples were purchased from different manufacturers from Austria and Germany.

\section{Instrumentation}

All standard solutions, tea samples and SPE fractions were determined using a 1100 Series HPLC Value System from Agilent (Santa Clara, USA) equipped with a 1100 Series variable wavelength detector (VWD). Analyses were performed using an ACE C18-PFP $(5 \mu \mathrm{m}, 4.6 \times 150 \mathrm{~mm}$, Advanced Chromatography Technologies Ltd, Aberdeen, Scotland) analytical column. Mobile phase was a composition of $0.1 \%$ TFA in water (eluent $\mathrm{A}$ ) and $\mathrm{MeOH}$ (eluent B). HPLC measurements were accomplished by a delayed gradient elution. The gradient program was performed using following steps (min/\% eluent B): 0/5, 1/5, 28/63, 30/90, $32 / 90,35 / 5,40 / 5$. The flow rate was $1 \mathrm{~mL} \mathrm{~min}^{-1}$ and the injection volume was $20 \mu \mathrm{L}$. Temperature of the column oven was set to $40{ }^{\circ} \mathrm{C}$ and detection of tea samples and caffeine was performed at $280 \mathrm{~nm}$.

Attenuated total-reflection infrared spectroscopy (ATRIR) measurements of the polymers were executed on a Spectrum 100 FT-IR Spectrometer (Perkin Elmer, Waltham, USA) with a diamond measurement cell and a ZnSe crystal as focusing element. For each spectrum, ten scans were performed in the range between 4000 and $650 \mathrm{~cm}^{-1}$ at a resolution of $4 \mathrm{~cm}^{-1}$. For nitrogen sorption porosimetry a Quantachrome Nova Station 200 (Quantachrome Instruments, Boynton Beach, FL-USA) was employed. Adsorption studies were executed on a ThermoMixer $\mathrm{C}$ from Eppendorf (Hamburg, Germany). Separation of supernatants was performed by a Centrifuge 5418 from Eppendorf. 


\section{Preparation of Standard Solutions}

Standard solution of caffeine $\left(1000 \mu \mathrm{g} \mathrm{mL}^{-1}\right)$ was prepared in water. This stock solution was stored at $4{ }^{\circ} \mathrm{C}$ in the dark. Working standard solutions were daily prepared by diluting the stock solution with water.

\section{Extraction and Analysis of Tea Samples}

$1 \mathrm{~g}$ of tea was extracted with $15 \mathrm{~mL}$ water in an ultrasonic bath at $70{ }^{\circ} \mathrm{C}$ for $15 \mathrm{~min}$. After centrifugation the supernatant was collected. To ensure a complete extraction of caffeine, each sample was extracted for three times and the supernatants were combined in a $50 \mathrm{~mL}$ volumetric flask. Extracts were then diluted with water to $50 \mathrm{~mL}$ filtered using H-PTFE filters and transferred into glass vials for quantification by HPLC-UV.

\section{Polymer Synthesis}

Poly(GMA/EGDMA) particles were prepared by a thermally initiated polymerization procedure (Fig. 1). Briefly, $4.32 \mathrm{~mL}$ (33 mmol) GMA, $7.10 \mathrm{~mL}$ (38 mmol) EGDMA and $0.3 \mathrm{~g}$ AIBN $(2 \mathrm{mmol}$ ) were dissolved in $45 \mathrm{~mL} \mathrm{ACN}$ in a $100-$ $\mathrm{mL}$ round-bottomed flask. Then the solution was flushed with argon for $10 \mathrm{~min}$ to remove oxygen. Afterwards, the flask was equipped with a condenser, placed in an oil bath and polymerization was performed at $60{ }^{\circ} \mathrm{C}$ for $16 \mathrm{~h}$ under stirring. The resulting polymer was ground using mortar and pestle and thoroughly washed with water and $\mathrm{MeOH}$. Finally, the polymer particles were dried at $50{ }^{\circ} \mathrm{C}$.
Histidine Functionalization of Poly(GMA/EGDMA)

\section{Particles}

For modification of poly(GMA/EGDMA), $1 \mathrm{~g}$ of sorbent was mixed with $25 \mathrm{~mL} 2 \mathrm{M}$ sodium carbonate solution in a $100-\mathrm{mL}$ round-bottomed flask equipped with a condenser followed by addition of $25 \mathrm{~mL}$ saturated L-histidine monohydrochloride monohydrate. Functionalization was then executed at $70{ }^{\circ} \mathrm{C}$ for $16 \mathrm{~h}$ under continuous stirring. The resulting polymer was finally washed with water and $\mathrm{MeOH}$ and dried at $45^{\circ} \mathrm{C}$ under vacuum.

\section{Adsorption Isotherms}

Determination of the polymer capacity was accomplished by several batch adsorption experiments. Therefore 10-12 mg of polymer was placed into $2 \mathrm{~mL}$ reaction tubes followed by addition of $1 \mathrm{~mL}$ standard solution at concentrations ranging from 25 to $250 \mu \mathrm{g} \mathrm{mL}^{-1}$. Then the tubes were placed in a thermo-mixer and incubated at room temperature for $1 \mathrm{~h}$ and $1200 \mathrm{rpm}$. Afterwards centrifugation was performed for $3 \mathrm{~min}$ at $14,000 \mathrm{rpm}$. Finally, the supernatants were filtered using H-PTFE filters and analyzed by HPLC-UV.

\section{Adsorption Kinetics}

Kinetic parameters of the adsorption process were determined in additional adsorption experiments. $10-12 \mathrm{mg}$ of polymer was placed in $2 \mathrm{~mL}$ reaction tubes and $1 \mathrm{~mL}$ of a $100 \mathrm{mg} \mathrm{L}^{-1}$ caffeine standard was added. Adsorption was then performed using a thermo-mixer at room temperature and $1200 \mathrm{rpm}$. Reactions were stopped at different time intervals ranging from 5 to $90 \mathrm{~min}$. After centrifugation for $3 \mathrm{~min}$ at $14,000 \mathrm{rpm}$ the supernatants were filtered using H-PTFE filters and analyzed by HPLC-UV.
Fig. 1 A Reaction scheme for synthesis of (c) poly(GMA/ EGDMA) by copolymerization of (a) glycidyl methacrylate and (b) ethylene glycol dimethacrylate. Reaction $\mathbf{B}$ shows the functionalization with histidine (d)

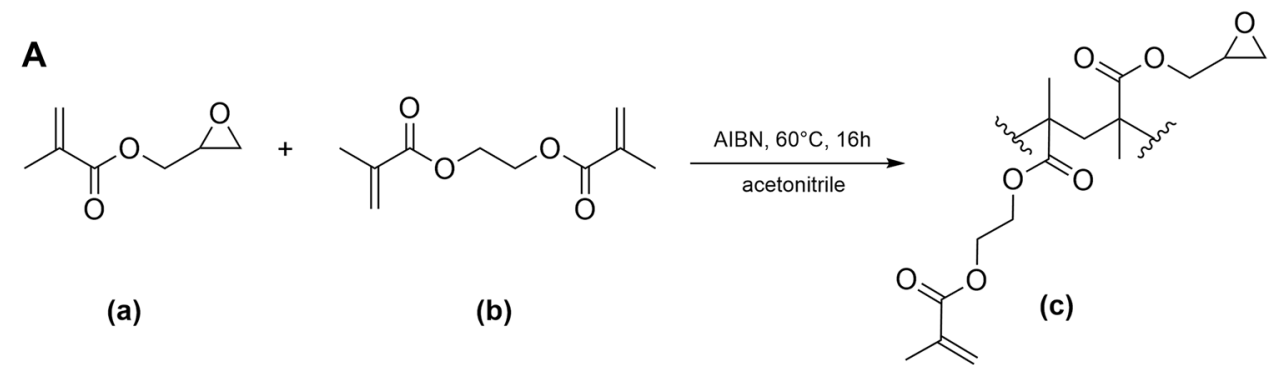

B<smiles>CC(=O)OCC1CO1</smiles>

(c)<smiles>N[C@@H](Cc1c[nH]cn1)C(=O)O</smiles>

(d)<smiles>CC(C)C(=O)OCC(O)CNC(Cc1c[nH]cn1)C(=O)O</smiles>

(e) 


\section{Clean-up of Tea Samples/SPE Procedures}

Synthesized polymers were tested for isolation of caffeine from different tea samples. First, $30 \mathrm{mg}$ of sorbent was packed into $1 \mathrm{~mL}$ SPE cartridges with polyethylene frits above and below the sorbent bed. For conditioning $1 \mathrm{~mL}$ $\mathrm{MeOH}$ was applied to the cartridge followed by loading $0.5 \mathrm{~mL}$ of diluted tea extract $1: 19(\mathrm{v} / \mathrm{v})$. After that elution of caffeine was executed using $3 \mathrm{~mL}$ of Milli-Q water. Solutions were finally quantified by HPLC-UV. Clean-up efficiency was further compared to the commercial available sorbent Oasis HLB. The Generic SPE protocol was obtained from Waters Corp [20]. Conditioning of the sorbent was executed using $1 \mathrm{~mL} \mathrm{MeOH}$ followed by $1 \mathrm{~mL}$ Milli-Q water. Then $0.5 \mathrm{~mL}$ of diluted tea extract 1:19(v/v) was applied to the polymer and subsequently washed with $1 \mathrm{~mL} \mathrm{5 \%} \mathrm{MeOH}$ in water (v/v). Finally, elution was performed with $1 \mathrm{~mL}$ $\mathrm{MeOH}$. Eluted fractions were evaporated to dryness, redissolved in $0.5 \mathrm{~mL}$ Milli-Q water and transferred to HPLC-UV analysis. All experiments were carried out in triplicate.

\section{Method Validation}

Performance evaluation of the HPLC method developed for determination of caffeine was accomplished according to international guidelines [21, 22]. The following parameters were determined: linearity, repeatability, accuracy, instrumental limits, method precision and stability of analytes.

Linearity was examined by measuring a standard solution (see "Preparation of Standard Solutions") at concentrations between 25 and $200 \%$ of target concentration $\left(20 \mu \mathrm{g} \mathrm{mL}^{-1}\right)$. For determination of the repeatability, a standard solution containing the target concentration level was prepared. Ten replicates per day were measured and repeated on three consecutive days to examine the intra-day and inter-day repeatability. Accuracy was investigated by spiking tea extracts with caffeine standard solutions at three different concentrations. Limit of detection (LOD) and limit of quantitation (LOQ) were determined from a calibration curve at concentrations ranging from 0.01 to $0.1 \mu \mathrm{g} \mathrm{mL}^{-1}$. Calculations of instrumental limits were executed referring to DIN32645: 2008-11 (Eqs. 1 and 2).

$$
\begin{aligned}
& \mathrm{LOD}=\frac{s_{y . x}}{b} \cdot t_{1-\alpha, v} \cdot \sqrt{\frac{1}{N}+\frac{1}{n}+\frac{\bar{x}^{2}}{\sum_{i=1}^{N}\left(x_{i}-\bar{x}\right)^{2}}} \\
& \mathrm{LOQ}=k \cdot \frac{s_{y . x}}{b} \cdot t_{1-\alpha, v} \cdot \sqrt{\frac{1}{N}+\frac{1}{n}+\frac{(\mathrm{LOQ}-\bar{x})^{2}}{\sum_{i=1}^{N}\left(x_{i}-\bar{x}\right)^{2}}}
\end{aligned}
$$

$s_{y . x}$ is the residual standard deviation of $y$ around the regression line, $b$ is the slope, $t_{1-\alpha, v}$ presents the student factor for $95 \%$ one sided for LOD and two sided for LOQ, $N$ is the number of calibration points and $n$ being the number of repetitions. Furthermore, the squared mean of x-values $\bar{x}^{2}$ and $\sum_{i=1}^{N}\left(x_{i}-\bar{x}\right)^{2}$ were calculated. The LOQ was iteratively determined. As recommended by DIN32645: 2008-11, $k$ was set to 3. Convergences of LOQ quickly occurred and the result was taken when the percental change from one iteration step to the next was no more significant [23].

Degradation of employed analytes in standard solution was studied over 14 consecutive days. Therefore, the standard solution was stored at $4{ }^{\circ} \mathrm{C}$ in the dark and the amount of analyte was determined daily.

\section{Results and Discussion}

\section{Polymer Composition and Characterization}

Isolation of leading compounds from plant matrices requires sorbent types with appropriate functional groups for interaction. Often mixed-mode materials with ion-exchange properties are used. These materials are typically crosslinked with DVB leading to a hydrophobic backbone. However, previous studies demonstrated that sorbents crosslinked with EGDMA as hydrophilic compound also exhibit excellent results for application with plant extracts [24, 25]. The functional modifier HIS was chosen as it contains anionic (imidazole) and cationic (carboxylic acid) ion-exchange moieties.

ATR-IR spectra of the dried functionalized polymer as well as the monomer, crosslinker and HIS were recorded (Fig. S1). The strong and sharp band at $1720 \mathrm{~cm}^{-1}$ can be assigned as the $\mathrm{C}=\mathrm{O}$ asymmetric stretch vibration. At $1637 \mathrm{~cm}^{-1}$ the $\mathrm{C}=\mathrm{C}$ stretch vibration of the methacrylate residues can be found in the spectra for GMA and EGDMA but significantly decreased after polymerization. Especially for GMA, the band at $1254 \mathrm{~cm}^{-1}$ representing the asymmetric stretch vibration of the epoxide ring is visible. A distinct band for the $\mathrm{C}-\mathrm{O}-\mathrm{C}$ stretch vibration of the ester of GMA and EGDMA can be assigned at $1143 \mathrm{~cm}^{-1}$. Due to broader bands in the polymer spectrum and thus overlapping of weak HIS signals no distinct functionalization can be confirmed by the ATR-IR spectra. However, differences between a ringopened polymer and HIS-functionalized polymer got visible in solid-phase extraction experiments.

Additionally, nitrogen adsorption porosimetry was executed to investigate specific surface areas of the synthesized sorbent. For the HIS-modified polymer, experiments revealed areas of $92 \mathrm{~m}^{2} \mathrm{~g}^{-1}$ whereas the non-modified polymer showed a slightly lower area of around $86 \mathrm{~m}^{2} \mathrm{~g}^{-1}$. In addition, batch-to-batch reproducibility of polymer modification was checked for three batches of polymers and showed only slightly deviations $<5 \%$. 


\section{Adsorption Capacity}

To investigate the affinity of the synthesized polymers for caffeine, several adsorption experiments were executed. Different concentrations of caffeine standard solutions in water were incubated together with fixed amounts of polymer to determine the corresponding binding isotherms (Fig. 2). The adsorbed amount $q$ of caffeine was calculated by following equation:

$q=\frac{\left(c_{\mathrm{i}}-c_{\mathrm{e}}\right) \cdot V}{m}$

where $c_{\mathrm{i}}$ and $c_{\mathrm{e}}$ represent the concentrations of caffeine at the initial and equilibrium, respectively. $V$ is the volume of standard solution used for the experiment and $m$ is the dry mass of the applied polymer.

Experiments showed a distinct correlation between loading capacity and concentration of standard solutions where increasing concentrations resulted into higher adsorption of caffeine. Maximum binding capacities were calculated using the Langmuir adsorption model. Calculation of model parameters was executed using the following equation:

$q=\frac{K_{\mathrm{L}} \cdot q_{\mathrm{m}} \cdot c}{1+K_{\mathrm{L}} \cdot c}$

where $q_{\mathrm{m}}$ represents the maximum capacity, $K_{\mathrm{L}}$ the Langmuir coefficient, $c$ the concentration of standard solution and $q$ the loaded amount of caffeine. For the HIS-modified material, a maximum capacity of $3.01 \mathrm{mg} \mathrm{g}^{-1}$ polymer could be obtained. Additionally the non-modified polymer was measured and revealed a higher capacity of $4.82 \mathrm{mg}$

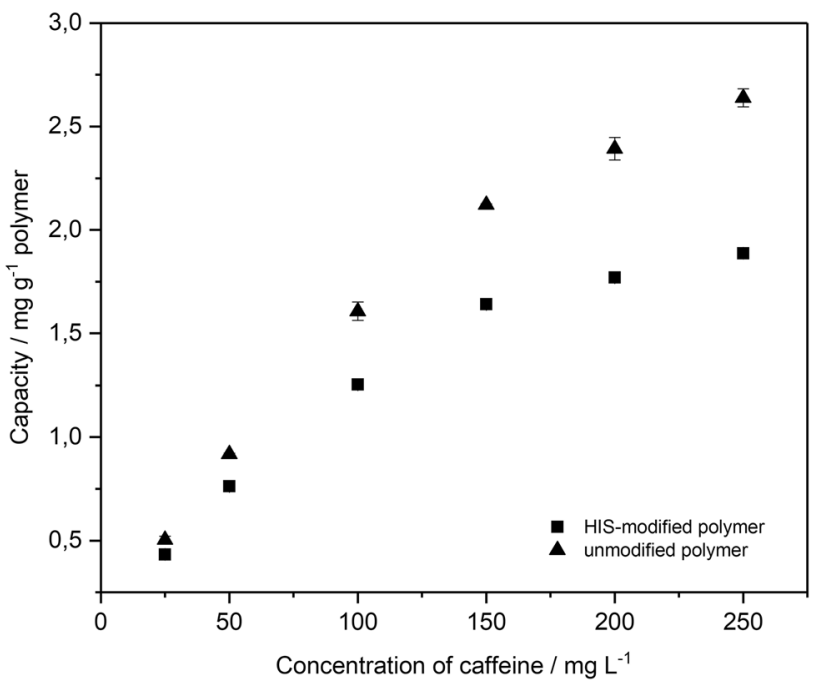

Fig. 2 Adsorption isotherms for caffeine on histidine-modified and non-modified polymer $(n=3)$. Standard deviations are represented as error bars $\mathrm{g}^{-1}$ polymer. These results indicate that modification of the polymer backbone leads to slightly lower capacity.

\section{Adsorption Kinetics}

For better understanding of the process kinetics, additional batch adsorption experiments with the HIS-modified and non-modified polymer were executed. Results of these experiments are shown in Fig. 3. As mentioned before, the kinetic experiments confirm a higher adsorption of caffeine on the non-modified polymer. For the evaluation of the adsorption kinetics, two kinetic models were used. The pseudo-first-order rate expression of the Lagergren model is expressed using following equation:

$q_{t}=q_{e} \cdot e^{-k_{1} \cdot t} \cdot\left(e^{k_{1} \cdot t}-1\right)$

where $t$ represents the rebinding time, $q_{t}$ the adsorption capacity at different time intervals, $q_{e}$ the adsorption capacity at equilibrium and $k_{1}$ the first-order rate constant. Furthermore a pseudo-second-order model was tested using following equation:

$q_{t}=\frac{k_{2} \cdot t \cdot q_{e}^{2}}{k_{2} \cdot t \cdot q_{e}+1}$

where $k_{2}$ is the rate constant of the second-order adsorption.

Data show that adsorption occurred quickly on both polymers and flattens after $15 \mathrm{~min}$. Fitting of the two kinetic models revealed that for the HIS-modified polymer $\left(R^{2}=0.934\right)$ and for the non-modified polymer the pseudo-second-order fits better $\left(R^{2}=0.936\right)$. Furthermore,

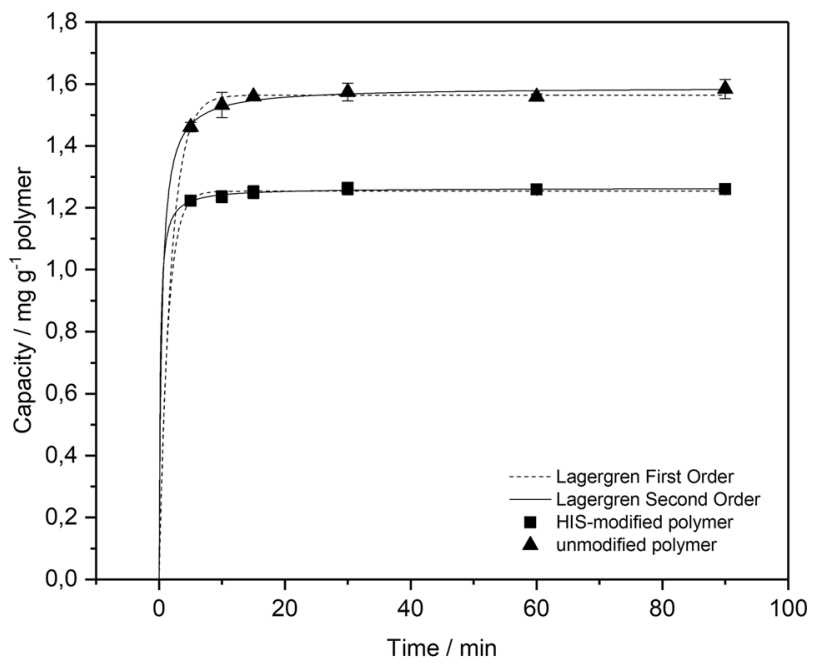

Fig. 3 Adsorption time profiles for caffeine on histidine-modified and non-modified polymer at different time intervals $(n=3)$. Standard deviations are represented as error bars 
rate constants were calculated for both models and showed for the HIS-modified polymer that $k_{2}$ is $4.50 \mathrm{~g} \mathrm{mg}^{-1} \mathrm{~min}^{-1}$ and for the non-modified polymer a $k_{2}$ value of $1.52 \mathrm{~g}$ $\mathrm{mg}^{-1} \min ^{-1}$ was observed.

\section{Clean-up and Analysis of Tea Samples}

In this work, we focused on the development of a simple sample preparation procedure using SPE. Previous studies successfully showed that implication of weak anion-exchange-functionalized materials demonstrate high potential for isolation and clean-up of crude plant extracts [24, 25]. After addition of HIS onto the present polymer backbone, the carboxy and imidazole functionalities remain free for interaction with molecules in solution. The imidazole ring of histidine is aromatic at all $\mathrm{pH}$

Table 1 Collection of black and green tea samples with their corresponding content of caffeine and recovery rates after optimized solidphase extraction $(n=3)$

\begin{tabular}{lll}
\hline Sample & $\begin{array}{l}\text { Caffeine content }\left(\mathrm{mg} \mathrm{kg}^{-1}\right) \\
\text { tea }^{\mathrm{a}}\end{array}$ & Recovery $^{\mathrm{b}}(\%)$ \\
\hline BT-12 & 28.4 & 92.9 \\
BT-13 & 31.6 & 90.0 \\
BT-23 & 28.7 & 91.4 \\
BT-63 & 16.1 & 88.7 \\
GT-1 & 18.9 & 89.9 \\
GT-2 & 21.4 & 90.8 \\
GT-3 & 16.8 & 91.0 \\
GT-4 & 18.4 & 89.5 \\
\hline
\end{tabular}

${ }^{\mathrm{a}} \mathrm{RSD}<2 \%$

${ }^{\mathrm{b}} \mathrm{RSD}<1 \%$

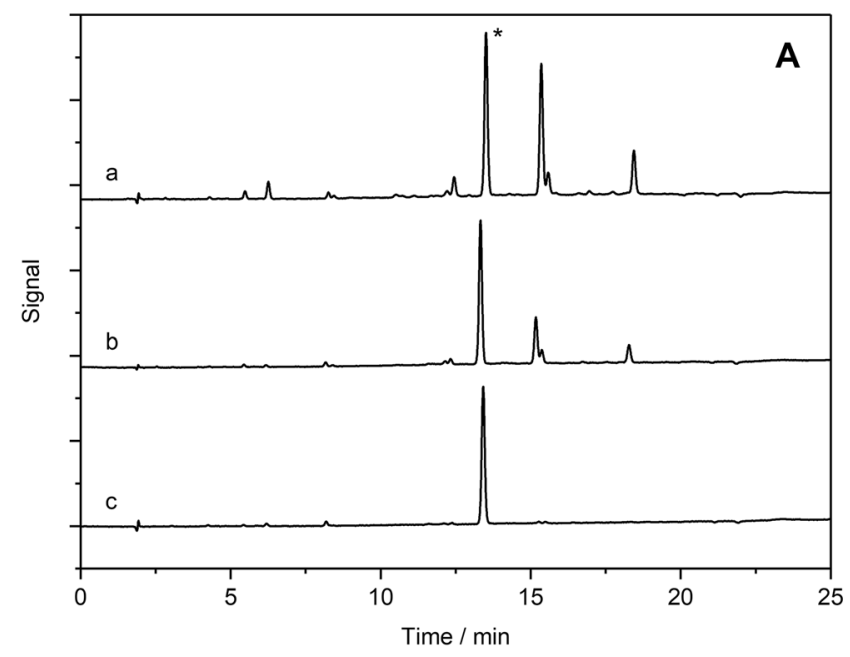

values [26] and contains six $\pi$-electrons where it can form $\pi$-stacking interactions with other aromatic compounds such as caffeine [27].

A first attempt using a classical SPE process with $\mathrm{MeOH}$ conditioning, loading of sample, water washing and elution with 20:80 ACN/water (v/v) resulted in low sample recoveries. Caffeine was mainly found in the wash solution throughout these experiments. In a next step, the process setup was changed by directly eluting caffeine from the sorbent without a prior washing step which yielded better results in comparison with the preliminary experiments. Nearly all interferences remained on the sorbent and an almost selective elution of caffeine with water was possible. We assume that caffeine is weakly interacting with the HIS residue of the polymer via $\pi-\pi$ interaction which allows easy elution by applying mild conditions. The optimized protocol was then employed for isolation and clean-up of eight black and green tea samples and achieved recoveries ranging from 89 to $93 \%$ (Table 1). Interfering peaks were notably reduced during the whole analysis. Figure 4 represents chromatograms of black and green tea extracts before and after SPE with the HIS-modified polymer and the unmodified polymer where just the epoxid ring was opened. Experiments with the control polymer, where only base-catalyzed ring opening of the epoxid of GMA was performed, showed lower recoveries (80-83\%) and considerably more interferences.

Clean-up of the applied tea extracts was further tested with Oasis HLB as commercial available sorbent and the corresponding generic SPE protocol. Chromatograms after clean-up revealed more interfering signals in comparison to the SPE procedures with the HIS-modified polymer (Fig. S2). However, recoveries were slightly higher ranging from 96 to $98 \%$. These results indicate a better clean-up

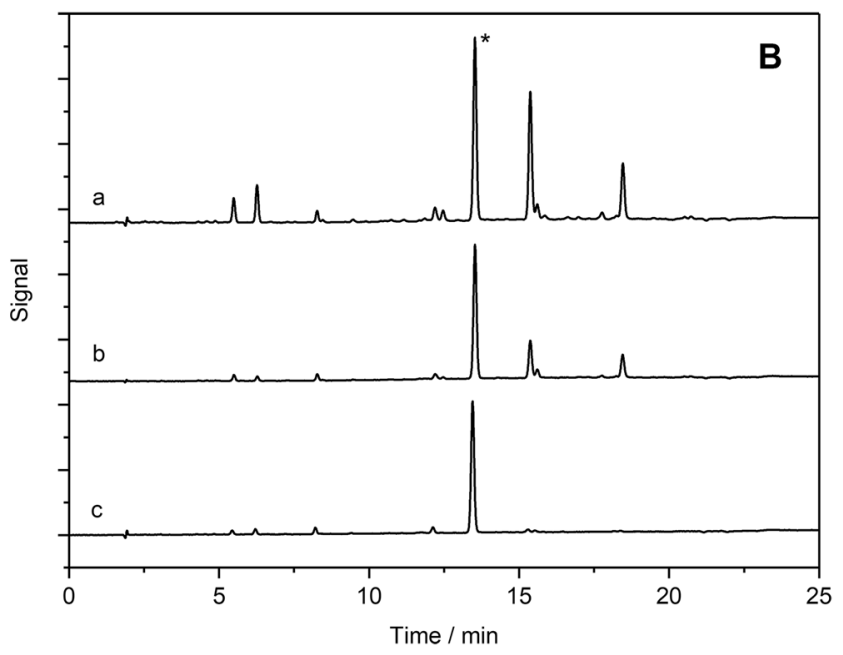

Fig. 4 Chromatograms of A green tea and B black tea extracts (a) before and after application of the proposed SPE procedure with (b) unmodified and (c) HIS-modified polymer. The marked peak represents caffeine. Chromatograms are plotted with same dilution factors 
Table 2 Performance evaluation of the applied analytical method

\begin{tabular}{|c|c|c|c|c|c|}
\hline \multicolumn{2}{|l|}{ Linearity } & \multicolumn{2}{|l|}{ Repeatability } & \multicolumn{2}{|l|}{ Limits } \\
\hline Regression equation & $R^{2}$ & $\begin{array}{l}\text { Intra-day RSD } \\
(\%)\end{array}$ & $\begin{array}{l}\text { Inter-day RSD } \\
(\%)\end{array}$ & $\begin{array}{l}\text { LOD } \\
\left(\mathrm{ng} \mathrm{mL}^{-1}\right)\end{array}$ & LOQ $\left(n g L^{-1}\right)$ \\
\hline$y=30.595 x-2.9358$ & 0.9999 & $0.06-0.10$ & 0.10 & 6.10 & 19.20 \\
\hline
\end{tabular}

Table 3 Accuracy of the proposed method for extracts spiked with caffeine standard solution at three concentration levels $(n=3)$

\begin{tabular}{lcl}
\hline Added standard $\left(\mu \mathrm{g} \mathrm{L}^{-1}\right)$ & Recovery $(\%)$ & RSD $(\%)$ \\
\hline 10 & 99.5 & 0.89 \\
20 & 100.5 & 0.76 \\
30 & 101.0 & 0.11 \\
\hline
\end{tabular}

efficiency for isolation of caffeine with the new sorbent modified with HIS.

\section{Validation Parameters}

Suitability of the proposed SPE method was determined by calculation of important validation parameters (Table 2). Caffeine concentrations from 25 to $200 \%$ of target concentration $\left(20 \mu \mathrm{g} \mathrm{mL}^{-1}\right)$ showed good linearity with a regression coefficient of 0.9999. Obtained LOD and LOQ values calculated by equations referring to DIN were 6.10 and $19.20 \mathrm{ng} \mathrm{mL}^{-1}$, respectively. Furthermore, the intra-day $(n=10)$ and inter-day $(n=30)$ repeatability was examined and only showed minimal differences, verified by RSD values between 0.09 and $0.10 \%$. Additionally, the accuracy of the method was checked by spiking tea extracts with caffeine at three concentration levels. Recoveries and RSD values of accuracy measurements are presented in Table 3. HPLC analyses of caffeine standard solution over a time range of 14 days revealed no significant decrease of concentration $<99 \%$.

\section{Conclusion}

Functionalization of a poly(GMA/EGDMA)-based polymer with HIS as functional group demonstrated excellent isolation performance for caffeine. Due to the imidazole moiety of HIS, $\pi-\pi$ interactions between sorbent and analyte can be formed. The developed SPE protocol for black and green tea samples was able to remove nearly all interferences especially around the caffeine peak. Adsorption experiments showed good capacities for the synthesized sorbent and clear second-order kinetics for the modified and non-modified material. An extensive validation of the proposed method was also executed and confirmed the high applicability of the developed method.

Acknowledgements Open access funding provided by University of Innsbruck and Medical University of Innsbruck. Florian Meischl is recipient of a DOC Fellowship of the Austrian Academy of Sciences at the Institute of Analytical Chemistry and Radiochemistry, University of Innsbruck. We would like to thank Norbert Köpfle, MSc. (Institute of Physical Chemistry, University of Innsbruck) for determination of the specific surface areas.

\section{Compliance with Ethical Standards}

Conflict of Interest The authors declare that there are no conflicts of interest.

Ethical Approval This article does not contain any studies with human participants or animals performed by any of the authors.

Open Access This article is distributed under the terms of the Creative Commons Attribution 4.0 International License (http://creativeco mmons.org/licenses/by/4.0/), which permits unrestricted use, distribution, and reproduction in any medium, provided you give appropriate credit to the original author(s) and the source, provide a link to the Creative Commons license, and indicate if changes were made.

\section{References}

1. Kim HK, Verpoorte R (2010) Phytochem Anal 21:4-13

2. Huie CW (2002) Anal Bioanal Chem 373:23-30

3. Tekel J, Hatrík S (1996) J Chromatogr A 754:397-410

4. Hennion M-C (1999) J Chromatogr A 856:3-54

5. Andrade-Eiroa A, Canle M, Leroy-Cancellieri V, Cerdà V (2016) TrAC Trends Anal Chem 80:641-654

6. Augusto F, Hantao LW, Mogollón NG, Braga SC (2013) TrAC Trends Anal Chem 43:14-23

7. Harris DC (2014) Lehrbuch der quantitativen analyse. Springer, Berlin Heidelberg

8. Kellner R, Mermet J-M, Otto M, Valcarcel M, Widmer HM (2004) Analytical chemistry. Wiley, Weinheim

9. Tian M, Yan H, Row KH (2009) Anal Lett 43:110-118

10. Meischl F, Schemeth D, Harder M, Köpfle N, Tessadri R, Rainer M (2016) J Environ Chem Eng 4:4083-4090

11. Hussain S, Güzel Y, Schönbichler SA, Rainer M, Huck CW, Bonn GK (2013) Anal Bioanal Chem 405:7509-7521

12. Yongfeng K, Wuping D, Yan L, Junxia K, Jing X (2012) Carbohydr Polym 88:459-464

13. Pascault J-P, Williams RJJ (2010) Epoxy polymers. Wiley, Weinheim

14. Rainer M, Muhammad NuH, Huck CW, Feuerstein I, Bakry R, Huber LA, Gjerde DT, Zou X, Qian H, Du X (2006) Rapid Commun Mass spectrom 20:2954-2960 
15. O’Mahony L, Akdis M, Akdis CA (2011) J Allergy Clin Immunol 128:1153-1162

16. Schneider E, Rolli-Derkinderen M, Arock M, Dy M (2002) Trends Immunol 23:255-263

17. Sakuragi M, Tsuzuki S, Hasuda H, Wada A, Matoba K, Kubo I, Ito Y (2009) J Appl Polym Sci 112:315-319

18. Lee ES, Shin HJ, Na K, Bae YH (2003) J Control Release 90:363-374

19. Özcan AA, Say R, Denizli A, Ersöz A (2006) Anal Chem 78:7253-7258

20. Waters Corp (2008) Oasis sample preparation-application notebook

21. Shabir GA (2004) J Valid Technol 10:210-218
22. Shabir GA (2005) J Valid Technol 10:314-325

23. Kromidas S (2011) Handbuch Validierung in der Analytik. Wiley, Weinheim

24. Schemeth D, Noël J-C, Jakschitz T, Rainer M, Tessadri R, Huck CW, Bonn GK (2015) Anal Chimi Acta 885:199-206

25. Meischl F, Kirchler CG, Jäger MA, Huck CW, Rainer M (2017) J Sep Sci 41(3):704-712

26. Mrozek A, Karolak-Wojciechowska J, Kieć-Kononowicz K (2003) J Mol Struct 655:397-403

27. Wang L, Sun N, Terzyan S, Zhang X, Benson DR (2006) Biochemistry 45:13750-13759 